\title{
DIMENSIONAMENTO DE CARGA-HUMANA BASEADO NA EFICIÊNCIA DE PROFISSIONAIS TERCEIRIZADOS VERSUS PROFISSIONAIS VERTICALIZADOS
}

\section{WORKFORCE BALANCE BASED ON THE EFFICIENCY OF OUTSOURCED VERSUS INTERNAL WORKERS}

\author{
Diego Vinicius de Souza ${ }^{1}$; Tiago Pascoal Filomena ${ }^{2}$; Michel Jose Anzanello ${ }^{3}$; Francisco José \\ Kliemann Neto ${ }^{4}$ \\ ${ }^{1}$ Universidade Federal do Rio Grande do Sul - UFRGS - Porto Alegre -Brasil \\ vinicius@producao.ufrgs.br \\ ${ }^{2}$ The George Washington University - Washington DC - EUA \\ tiagopf@gwu.edu \\ ${ }^{3}$ RUTGERS - The State University of New Jersey - New Jersey - EUA \\ michelja@eden.rutgers.edu \\ ${ }^{4}$ Universidade Federal do Rio Grande do Sul - UFRGS - Porto Alegre -Brasil \\ kliemann@producao.ufgrs.br
}

\section{Resumo}

A opção pela utilização de recursos terceirizados ou verticalizados aparece como incógnita constante em setores de manutenção, principalmente nos segmentos que operam equipamentos de tecnologia especifica. Tal decisão acaba por impactar no número e especialidade dos profissionais alocados aos procedimentos. Este artigo apresenta uma sistemática simplificada para dimensionamento de carga-humana baseada em estudo de tempos e análise de eficiência. Para tanto, dados coletados em processos de manutenção são inicialmente organizados através da matriz relacionadora, a qual possibilita melhor entendimento acerca do tempo demandado por distintos perfis técnicos para execução dos procedimentos analisados. Tais resultados são então integrados a um indice de eficiência, o qual foi desenvolvido com base nos tempos requeridos por profissionais verticalizados e terceirizados para execução dos procedimentos. Fatores de disponibilidade relativos a profissionais verticalizados também são considerados neste índice. $O$ método proposto permite comparar o desempenho de equipes de manutenção verticalizadas frente a equipes terceirizadas em termos de performance e custo, servindo como ferramenta de suporte para contratações de funcionários, estruturação de editais de concursos públicos e gestão de contratos de terceirização. A aplicação do método em uma empresa de transporte urbano, TRENSURB S/A, apontou os recursos terceirizados como mais adequados para a manutenção da frota de trens.

Palavras-chave: Dimensionamento de carga-humana, eficiência, manutenção, terceirização. 


\section{Introdução}

Cenários altamente competitivos têm imposto severas condições para a sobrevivência das empresas, demandando esforços contínuos no aprimoramento dos processos produtivos. Os diferenciais competitivos, outrora centrados no preço e qualidade de um produto ou serviço (DE RON, 1998), têm se transferido para a capacidade de resposta às exigências do mercado, tornando imprescindível o desenvolvimento das competências da empresa (NARDI, 2004).

A definição de competência surgiu na década de 30 por intermédio de programas de treinamento e capacitação. Tais programas eram estruturados por supervisores e gerentes de empresas, e contaram com rápida difusão no contexto industrial por conta dos seus notáveis resultados (ODERICH, 2001). A gestão de competências busca a melhor alocação dos recursos produtivos, com vistas à elevação do patamar de desempenho do processo. Tal gestão depende de fatores relacionados ao mercado e de métodos de controle gerenciais, tornando-se altamente complexa na maioria das aplicações práticas (PINNINGTON; WOOLCOCK, 1995; TENG et al., 1995; POPPE; ZENGER, 1998; NARDI, 2004). Como conseqüência, percebe-se que diversas atividades não-centrais de uma organização, anteriormente desempenhadas por áreas de apoio da empresa, passaram a ser desempenhadas por equipes terceirizadas (PINNINGTON; WOOLCOCK, 1995; NETO, 1995).

Terceirização constitui-se na transferência da responsabilidade de execução de uma operação, serviço ou fase de um processo de uma empresa para outra (NETO, 1995; KRAJEWSKI; RITZMAN, 1999; ASKIN; GOLDBERG, 2002; McIVOR, 2008). De acordo com Belcourt (2006), a tercerização diferencia-se das tradicionais parcerias ou alianças por conta do fluxo dos serviços, os quais são executados exclusivamente no sentido provedor-usuário. De tal forma, não existe divisão de lucros ou contribuição mútua entre provedores e usuários. Hoecht and Trott (2006) acrescentam que a terceirização surgiu como estratégia para corte de custos nas funções periféricas da empresa, mas rapidamente alastrou-se para as funções vitais da organização.

Em termos de prestação de serviços, a terceirização apresenta como vantagens: $(i)$ redução de custos relativos à contratação e treinamento de funcionários, (ii) possibilidade de contratação de recursos altamente especializados, e (iii) maior flexibilidade no ajuste da força de trabalho frente a oscilações de demanda impostas pelo mercado (KRAJEWSKI; RITZMAN, 1999, GROSSMAN; HELPMAN, 2002; WEIDENBAUM, 2005). A possibilidade de substituição do provedor de determinado serviço, caso esse não se ajuste às necessidades específicas da empresa contratante, também figura como vantagem do sistema terceirizado (ESPINO-RODRIGUEZ; PADRÓROBAINA, 2005). Belcourt (2006) acrescenta que provedores de serviço terceirizado apóiam-se na 
oferta de recursos com elevados padrões de tecnologia, o que acaba por refletir nas características de qualidade do serviço oferecido. Uma comparação das estratégias, técnicas e benefícios da terceirização nos USA e Europa são reportadas por Kakabadse e Kakabadse (2002).

Em contrapartida, as limitações do sistema terceirizado são evidenciadas pela impossibilidade de se dispor pontualmente do serviço quando necessário, principalmente em setores onde a oferta de serviço especializado é reduzida em relação à demanda, ou em situações em que o provedor apresenta custos proibitivos à realidade da empresa contratante (KRAJEWSKI; RITZMAN, 1999; GROSSMAN; HELPMAN, 2005). Belcourt (2006) aponta que a substituição ou modificação de condições estabelecidas no início do contrato provedor-usuário também caracterizam-se como limitação, visto que acarretam elevação nos custos originalmente determinados. Em termos humanos, a utilização de recursos terceirizados impacta na moral dos trabalhadores pertencentes ao quadro funcional da empresa, os quais julgam-se menos habilitados a executar os procedimentos entregues à iniciativa terceirizada (BELCOURT, 2006). O aprendizado organizacional passa a ser aspecto fundamental nestas circunstâncias, visto que a utilização de recursos próprios da empresa ou a contratação e treinamento de pessoal para execução permanente das atividades surge como alternativa.

O aprendizado organizacional pode ser encarado como meio para a conquista de competências específicas no mercado. Cada trabalhador é visto como uma unidade básica do mecanismo de aprendizado dentro da organização, sendo a transferência do conhecimento para o restante do grupo responsabilidade do trabalhador e da empresa em que ele está inserido. Merha e Dhawan (2003) realizaram estudos em empresas indianas voltadas para o desenvolvimento de softwares, buscando analisar fatores que influenciassem o ambiente de aprendizado de membros de uma equipe de trabalhadores. Dentre vários fatores identificados, destacaram-se a flexibilidade dos meios de treinamento, saúde organizacional, interação entre trabalhadores e oportunidades para aprendizado. Segundo os autores, o conceito de empowerment também passou a ser expressivo no contexto de aprendizado organizacional, uma vez que as responsabilidades atribuídas aos trabalhadores refletem-se na qualidade dos procedimentos executados.

Agrell et al. (2002) apontam a dificuldade enfrentada pela gerência para difundir preceitos de melhorias e conhecimento de maneira eficiente através da estrutura verticalizada, principalmente em setores onde o processo depende da participação de vários trabalhadores. Para tanto, propuseram uma estrutura capaz de incentivar a transferência de conhecimento através dos vários níveis da força de trabalho. Tal estrutura está baseada em três premissas: aprender, ensinar e inovar. A estrutura complementa-se com a inclusão de programas de treinamento e divisão de lucros da empresa entre os trabalhadores. Nembhard e Uzumeri (2000a) alertam para a adequada escolha de um método 
para avaliação do aprendizado organizacional. Os métodos tradicionalmente utilizados agregam os trabalhadores sem levar em conta características particulares de cada membro em relação à aquisição de conhecimento, as quais possibilitariam descrições mais precisas do desempenho de cada trabalhador pertencente à estrutura verticalizada.

O processo de aquisição e disseminação de conhecimento tem sido estudado em várias perspectivas e com diferentes objetivos. Áreas relacionadas à Engenharia têm se focado na modelagem desses processos com o intuito de monitorar custos e horas de trabalho necessárias para execução de procedimentos em diversos setores (DAR-EL e RUBINOVITZ, 1991).

De acordo com MEADOR (1995), setores associados à manutenção de plantas produtivas constantemente avaliam os benefícios e limitações da utilização de recursos próprios (verticalizados) frente aos recursos terceirizados, bem como os impactos do aprendizado organizacional. Segundo o mesmo autor, existe uma relação direta entre o desempenho de uma planta e eficiência de seus serviços de manutenção. Tal eficiência está associada principalmente ao conhecimento dos operadores quanto à execução dos procedimentos demandados, o qual por sua vez depende dos programas de treinamento oferecidos e das sistemáticas de difusão de conhecimento adotadas pela gerência e operadores (AGRELL; BAGETOFT; TIND, 2002; MEHRA; DHAWAN, 2003).

De tal forma, a eficiência das atividades de manutenção apresenta-se como ponto crucial em diversas organizações, principalmente nas empresas que operam equipamentos de tecnologia específica. A pronta disponibilidade de um serviço e a qualidade com que o mesmo é executado, aliado a uma eficiente programação de manutenção, passam a figurar como fatores críticos para a competitividade e confiabilidade da empresa (BRETTHAUER; GAMALEJA; NEUMANN; HOFFMAN, 1998).

Por conta das diretrizes estratégicas vigentes na década de 1990, a TRENSURB S/A optou por terceirizar grande parte de suas atividades de manutenção (originalmente realizadas por colaboradores internos) com a finalidade de otimizar sua estrutura funcional. Atualmente, seguindo um direcionamento do governo federal, avalia-se a alternativa de novamente verticalizar as atividades manutentoras. Verifica-se, no entanto, a carência de sistemáticas que viabilizem o dimensionamento de carga-humana para as atividades de interesse levando em conta aspectos de custo e que, complementarmente, permitam a comparação da eficiência de profissionais terceirizados e verticalizados.

O presente artigo descreve um método simplificado para o dimensionamento de operadores relacionando perfís técnicos à eficiência da operação realizada, baseado em estudo de tempos e análise de eficiência. A sistemática é constituída pelas seguintes etapas: (i) Concepção da matriz 
relacionadora, a qual vincula as diversas atividades aos tempos demandados para sua execução; (ii) Avaliação de eficiência, a qual baseia-se no desenvolvimento de um índice de performance comparativo entre os recursos terceirizados e verticalizados; (iii) Cálculo das demandas de manutenção e Dimensionamento de pessoal; e (iv) Comparativo econômico entre verticalização e terceirização. A metodologia proposta é ilustrada em uma empresa ferroviária de transporte público.

\section{Descrição da sistemática proposta e exemplo numérico}

Nesta seção são descritos os passos da metodologia sugerida. Para uma maior clareza, dados numéricos são apresentados em paralelo aos procedimentos propostos.

\subsection{Concepção da matriz relacionadora}

Dados referentes à manutenção corretiva e preventiva foram coletados no Setor de Manutenção Leve (SEMLE) e no Setor de Oficina (SEOFI), respectivamente. Neste estudo, apresenta-se somente a análise realizada no SEMLE. Os procedimentos de manutenção e divisão das atividades foram inicialmente avaliados e possibilitaram a identificação dos perfis técnicos responsáveis pelas atividades (eletricistas, mecânicos e auxiliares, entre outros). Os tempos demandados para execução dos procedimentos (realizados em sua maioria por profissionais terceirizados) foram coletados diretamente do chão-de-manutenção. Os dados de tempo coletados foram então comparados com o banco de dados da empresa, assegurando-se assim a consistência das informações coletadas. Um exemplo simplificado do arranjo de tais informações é apresentado na Tabela 1, a qual traz a matriz relacionadora para manutenção do tipo $A L_{1}$. 
Tabela 1 - Matriz Relacionadora para manutenção $A L_{1}$

\begin{tabular}{lccc}
\hline \multirow{2}{*}{ Atividade } & \multicolumn{3}{c}{ Tempo de atividade por perfil técnico (h) } \\
\cline { 2 - 4 } & Eletricista & Mecânico & Auxiliares \\
\hline Limpeza e Inspeção - Caixa de chave de linha & 4 & & \\
Limpeza e Inspeção - Controlador principal & 3 & & \\
Limpeza e Inspeção - Caixa de partida do MA & 1 & 12 & 12 \\
Execução das máquinas de porta & & 4 & \\
Verificação bolsas de ar/freio/altura engates & & 4 & \\
\hline
\end{tabular}

Fonte: Relatório final TRENSURB S/A (2004)

A funcionalidade da matriz relacionadora está na praticidade de análise dos dados nela inseridos, uma vez que o somatório das colunas apresenta a carga-humana total (em horas) demandada por cada perfil técnico, enquanto que o somatório das linhas traz a carga horária demanda pelas atividades. Por exemplo, são necessárias 8 horas do perfil técnico "Eletricista" em uma manutenção do tipo $A L_{1}$. Matrizes semelhantes foram elaboradas para todas as atividades avaliadas.

\subsection{Avaliação de Eficiência}

Os procedimentos de manutenção da TRENSURB S/A são executados tanto por equipes terceirizadas quanto por equipes verticalizadas. Dados empíricos de performance denotam um desequilíbrio entre a eficiência da mão-de-obra terceirizada e a apresentada pelos colaboradores internos (verticalizados) da empresa. Essa discrepância, de acordo com a experiência dos gestores, indica que 1,6 funcionários verticalizados são necessários para equivaler ao desempenho de um colaborador terceirizado. Sendo este um dado empírico, realizou-se um comparativo de eficiência com a finalidade de calibrar esse indicador e, desta forma, ajustar o dimensionamento da cargahumana.

Inicialmente, propõe-se estimar a indisponibilidade média anual dos colaboradores verticalizados da empresa (definida como parâmetro $k$ ). O parâmetro $k$ é composto por dois componentes: $(i)$ o primeiro componente é gerado pelo somatório dos dias previstos para a ausência do colaborador (férias, atestado e prêmio), e então dividido pelo total de dias computados para desempenho de atividades profissionais (carga horária anual). Para o caso em análise, obteve-se um valor de 15\%. (ii) O segundo componente é representado por dados históricos de absenteísmo (medidos pelo departamento de recursos humanos da empresa), sendo estes da ordem de $2 \%$. A 
soma dos dois componentes aponta $17 \%$ como estimativa da indisponibilidade média de cada colaborador verticalizado de manutenção. Em relação ao profissional terceirizado, considera-se que o mesmo é remunerado por atividade desempenhada e sem vínculo empregatício com a empresa contratante. Por conta disso, não são avaliados índices de afastamentos.

A análise comparativa de eficiência entre os operadores terceirizados e verticalizados baseia-se na coleta dos tempos de execução dos procedimentos referentes à manutenção do tipo $A$ efetuada por duas equipes (uma terceirizada e outra verticalizada). Ambas as equipes são compostas pelo mesmo número de operadores e presumidamente dotadas de patamares semelhantes de conhecimento em torno da execução dos procedimentos. Os experimentos contaram com 5 repetições para cada equipe, sendo os resultados finais apresentados na Tabela 2.

Tabela 2 - Comparativo de eficiência em manutenções do tipo $A$

\begin{tabular}{lcc}
\hline & $\begin{array}{c}\text { Profissional } \\
\text { Verticalizado }\end{array}$ & $\begin{array}{c}\text { Profissional } \\
\text { Terceirizado }\end{array}$ \\
\hline $\begin{array}{l}\text { Número de funcionários } \\
\text { Tempo médio de execução } \\
\text { do procedimento (h) }\end{array}$ & 3 & 3 \\
Hora/Homem & 3,05 & 2,04 \\
\hline
\end{tabular}

Fonte: Relatório final TRENSURB S/A (2004)

A razão Hora/Homem entre profissionais verticalizados e terceirizados é aproximadamente $1,5(=9,15 / 6,12)$, passando a ser definida como relação de eficiência $(m)$ e permitindo comparar diretamente o desempenho dos dois grupos de profissionais. Sendo $k$ a taxa de indisponibilidade de um colaborador verticalizado da TRENSURB S/A, obtém-se o coeficiente de ineficiência do sistema, $I$, através da equação (1). $I>1$ denota cenários em que a utilização de profissionais terceirizados é vantajosa em termos de eficiência, justificando a utilização do termo "ineficiência" em relação aos operadores verticalizados.

$$
I=k+m=0,17+1,50=1,67
$$

O resultado da equação (1) indica que um trabalhador verticalizado demanda, em média, $67 \%$ a mais de tempo do que um operador terceirizado para execução da manutenção tipo $A$; tal coeficiente será usado como multiplicador dos somatórios de horas envolvidas em cada perfil 
técnico ao comparar-se mão-de-obra terceirizada e verticalizada para aquele tipo de manutenção. Vale acrescentar que $I$ deve ser estimado para todas as modalidades de manutenção executadas (descritas na sequência).

\subsection{Cálculo das demandas de manutenção e dimensionamento de pessoal}

Os tipos de manutenção executados na empresa estão baseados em intervalos de quilometragem $(P)$ cumpridos pelos trens, conforme apresentado na Tabela 3. A equação (2) possibilita estimar o número de manutenções do tipo $i\left(N_{i}\right)$ a serem realizadas em um período de tempo $(M)$. Considerando-se que a empresa possui 24 trens em atividade $\left(N_{t}\right)$ e que a quilometragem média anual por trem $(K)$ é $120.000 \mathrm{~km}$, estima-se que 8 manutenções do tipo $A L$ devem ser efetuadas mensalmente.

Tabela 3 - Tipos de manutenção avaliadas

\begin{tabular}{lc}
\hline \multicolumn{1}{c}{ Tipo de manutenção } & $\begin{array}{c}\text { Intervalo de } \\
\text { quilometragem }(\boldsymbol{P})\end{array}$ \\
\hline Tipo $A$ & 3.000 \\
Tipo $A L$ & 33.000 \\
Tipo $D$ & 100.000 \\
\hline Fonte: Relatório final TRENSURB S/A (2004) & \\
$N_{A L}=\left\lceil\frac{N t \times K}{M \times P}\right\rceil=\left\lceil\frac{24 \times 120.000}{12 \times 33.000}\right\rceil=8$
\end{tabular}

De maneira semelhante, estimaram-se 80 manutenções mensais do tipo $A$ e 3 manutenções mensais do tipo $D$.

O cálculo do número de profissionais verticalizados dos diversos perfis necessários para execução das manutenções $\left(N_{P E R F I L}\right)$ pode ser obtido por intermédio da equação (3). Salienta-se que os bancos de dados da TRENSURB S/A estão baseados em tempos de execução gerados por recursos terceirizados, visto que tais manutenções eram realizadas por profissionais desta modalidade, o que justifica a multiplicação pelo coeficiente de ineficiência $I$. Situações em que $I<1$ apontam cenários em que a utilização de profissionais verticalizados é vantajosa, conduzindo a um número inferior de trabalhadores quando comparado ao número de profissionais terceirizados 
necessários.

$$
N_{\text {PERFIL }}=\frac{N_{i} \times T}{D \times H} \times I
$$

onde $N_{i}$ é a demanda calculada por tipo de manutenção [da equação (2)], $T$ é o somatório de horas de cada tipo de manutenção (baseado em profissionais terceirizados), $D$ o número de dias por mês, $H$ a carga diária prevista por dia e $I$ o coeficiente de ineficiência do sistema. Considerando-se que um eletricista terceirizado demanda 84 horas para execução de uma manutenção do tipo $A L$ (informação oriunda do banco de dados da empresa) e que tal funcionário trabalha 8 horas/dia, 21 dias/mês, obtém-se uma estimativa de que são necessários aproximadamente 6 profissionais verticalizados com o perfil técnico Eletricista na execução de manutenção do tipo $A L$.

Tal sistemática de cálculo foi estendida para totalidade de perfis técnicos envolvidos e atividades manutentoras da empresa em questão, passando a constituir-se no principal instrumento para a tomada de decisões quanto a contratações de funcionários para o setor de manutenção. A sistemática proposta também é utilizada na estruturação de editais de concursos públicos e na gestão de contratos de terceirização.

\subsection{Comparativo econômico entre terceirização e verticalização}

A análise comparativa de custos associados por perfil técnico entre a estrutura verticalizada e a terceirizada conclui a sistemática proposta. Como exemplo, será analisado o perfil Eletricista, sendo que a mesma sistemática de cálculo foi estendida para os demais perfis técnicos.

O custo de um homem-hora terceirizado para o perfil eletricista é R \$16,77, de acordo com o banco de dados da TRENSURB S/A, sendo que a este valor não são incorporados encargos sociais ou benefícios. O profissional de mesmo perfil técnico associado à estrutura verticalizada incorre nos custos descritos na Tabela 4. 
Tabela 4 - Benefícios mensais de um eletricista verticalizado

\begin{tabular}{lc}
\hline \multicolumn{1}{c}{ Benefício } & R\$/mês \\
\hline Salário & 904,00 \\
Adic. Periculosidade & 181,40 \\
Férias (1/12) & 50,40 \\
$13^{\circ}$ Salário (1/12) & 50,40 \\
Encargos Sociais (39\% INSS e 8,5\% FGTS) & 322,58 \\
Vale Alimentação & 337,90 \\
Vale Transporte & 41,21 \\
Seguro Saúde & 3,50 \\
TOTAL & $\mathbf{1 . 9 0 1 , 3 9}$ \\
\hline
\end{tabular}

Fonte: Relatório final TRENSURB S/A (2004)

Considerando-se a disponibilidade da força-de-trabalho obtida na análise de eficiência, um colaborador verticalizado estaria disponível 139,5 horas das teóricas 168 horas previstas por mês. Dividindo-se o valor total obtido na Tabela 4 pelo número de horas disponíveis e multiplicando-se pelo coeficiente de ineficiência $I$, obtém-se R\$20,45 como valor hora-homem de um eletricista verticalizado, $22 \%$ superior ao valor-hora do profissional terceirizado. Salienta-se que esta comparação é baseada no salário de um funcionário em início de carreira. Com o passar dos anos, este funcionário terá sua remuneração aumentada em diversos períodos, onerando a estrutura de custos-fixos da empresa.

\section{Conclusão}

O presente estudo apresentou uma sistemática de dimensionamento de carga-humana para procedimentos de manutenção, com o objetivo principal de comparar a eficiência e custos de equipes de manutenção verticalizadas frente às terceirizadas.

Verificou-se que equipes internas (verticalizadas) da TRENSURB S/A apresentaram desempenho inferior aos profissionais terceirizados em termos de eficiência dos procedimentos de manutenção. De tal forma, faz-se necessária uma profunda análise na sistemática de execução das operações. O desenvolvimento de rotinas de treinamento dos funcionários internos e a padronização das atividades através de roteiros de manutenção são alternativas viáveis a serem implementadas, visando aumentar a eficiência do plantel interno e amenizar o déficit econômico em relação à terceirização. O sistema terceirizado também configura-se na alternativa mais adequada em termos 
econômicos ao considerar-se benefícios trabalhistas e disponibilidade funcional.

Apesar de estruturalmente simples, a sistemática de dimensionamento de carga-humana gerou resultados satisfatórios e coerentes quando avaliados pelos gestores da empresa. A matriz relacionadora permitiu associar de forma eficiente os perfis profissionais às cargas de tempo demandadas para execução dos procedimentos de manutenção. O desenvolvimento de um índice de eficiência concatenando $(i)$ tempos de execução de procedimentos por profissionais verticalizados e terceirizados e ( $i$ i) carga horária efetivamente disponível por profissionais verticalizados gerou uma forma verossímil de comparação de eficiência entre os dois enquadramentos profissionais. Sua utilização permitirá à empresa em estudo redimensionar a execução de suas atividades e promover melhorias no processo produtivo, bem como constituir-se-á em instrumento para a tomada de decisões gerenciais quanto à terceirização e incorporação de novos colaboradores ao quadro funcional.

Estudos futuros podem contemplar a performance de programas de treinamento sobre os colaboradores verticalizados da empresa através de modelagem por curvas de aprendizado (UZUMERI; NEMBHARD, 1998, ANZANELLO; FOGLIATTO, 2005, 2007). Outra sugestão consiste na utilização da mesma ferramenta para eficiente alocação de equipes de trabalhadores aos distintos procedimentos de manutenção, visando aumentar os indicadores de produção. As curvas de aprendizado seriam aplicadas em dados de performance coletados de trabalhadores verticalizados ao executarem procedimentos diversos. Tais procedimentos podem ser agrupados em famílias de distintas complexidades através de procedimentos de clusterização, o que reduziria o volume de dados a serem coletados. Os parâmetros gerados pela modelagem das curvas de aprendizado seriam então utilizados para identificar os trabalhadores mais propensos à execução das tarefas, de acordo com a duração e complexidade de tais tarefas.

\section{Referências}

AGRELL, P. J.; BOGETOFT, P.; TIND, J. Incentive Plans for Productive Efficiency, Innovation and Learning. International Journal of Production Economics, v. 78, n. 1, p.1-11, 2002.

cross ref

ANZANELLO, M.; FOGLIATTO, F. Alocação de modelos de produtos a equipes de trabalhadores através da modelagem de curvas de aprendizado. Revista Produção, v. 15, n. 02, p. 221-234, 2005.

cross ref

ANZANELLO, M.; FOGLIATTO, F. Learning curve modelling of work assignment in mass customized assembly lines. International Journal of Production Research, v. 45, p. 2919-2938, 2007.

cross

ASKIN, R; GOLDBERG, J. Design and Analysis of Lean Production Systems, New York, Ed. Wiley \& Sons, 2002. AUGUSTE, B.G.; HAO, Y.; WEIGAND, M. O lado do terceirizado: lucrando com os infra-serviços - HSM Management, v.8, n. 42, 2004. 
BELCOURT, M. Outsourcing - The benefits and the risks. Human Resource Management Review, v. 16, p. 269-279, 2006.

cross ${ }^{\text {ref }}$

BRETTHAUER, G.; GAMALEJA, T.; NEUMANN, U.; HOFFMAN, W. Integrated maintenance scheduling system for electrical energy systems. IEEE Transactions on Power Delivery, v. 13, n. 2, p. 655-660, 1998.

cross'

DAR-EL, E. M.; RUBINOVITZ, J. Using learning theory in assembly lines for new products, International Journal of Production Economics, v. 25, p. 103-109, 1991.

cross ref

DE RON, A. J. Sustainable Production: the Ultimate Result of a Continuous Improvement. International Journal of Production Economics, v. 56, p. 99-110, 1998.

cross ${ }^{\text {ref }}$

ESPINO-RODRIGUEZ, T., PADRÓ-ROBAINA, V. A resource-based view of outsourcing and its implications for organizational performance in the hotel sector. Tourism Management, v. 26, p. 707-721, 2005.

cross ref

GROSSMAN, G.; HELPMAN, E. Integration versus outsourcing in industry equilibrium. The Quarterly Journal of Economics, v. 117, n. 1, p. 85-120, 2002.

cross ref

GROSSMAN, G.; HELPMAN, E. Outsourcing in a global economy. Review of Economical Studies, v. 72, p. 135$159,2005$.

cross ref

HOECHT, A.; TROTT. P. Innovation risks of strategic outsourcing. Technovation, v. 26, p. 672-681, 2006.

cross ref

KAKABADSE, A; KAKABADSE, N. Trends in Outsourcing: contrasting USA and Europe. European Management Journal, v. 20, n. 2, p. 189-198, 2002.

cross ref

KRAJEWSKI, L.; RITZMAN, L. Operations Management, Strategy and Analysis, 5a edição. Editora AddisonWesley, 1999.

McIVOR, R. What is the right outsourcing strategy for you business? European Management Journal, v. 26, n. 1, p. 24-34, 2008.

cross ref

MEADOR, R.J. Maintaining the Solution to Operations and Maintenance Efficiency Improvement - PNL-SA -26005. Pacific Northwest National Laboratory, Richland, Washington, 1995.

MEHRA, K.; DHAWAN, S. K. Study of the Process of Organizational Learning in Software Firm in India. Technovation, v. 23, p. 121-129, 2003.

cross ref

MEHRA, K.; DHAWAN, S. K., Study of the process of organisational learning in software firm in India, Technovation, v. 23, p. 121-129, 2003.

cross ref

NARDI, K.C. Estruturação de dimensionamento de carga humana na área pública. 2004. Trabalho de Diplomação - Programa de Pós-Graduação em Engenharia de Produção - UFRGS, Porto Alegre.

NETO, J.A. Reestruturação industrial, terceirização e redes de subcontratação - Revista de Administração de Empresas, v.35, n. 2, p. 33-42, 1995. 
ODERICH, C.L. Gestão de competências gerenciais: noções e processos de desenvolvimento em três empresas gaúchas. 2001 - Dissertação. Universidade Federal do Rio Grande do Sul. Porto Alegre.

PEZERICO, L.A.M. Sistemas de Avaliação de Desempenho no Transporte Urbano: Uma abordagem para o setor metroferroviário. 2002 - Trabalho de Conclusão do Curso de Mestrado Profissionalizante em Engenharia de Produção. Porto Alegre.

PINNINGTON A., WOOLCOCK, P. How far is IS/IT outsourcing enabling new organizational structure and competences? International Journal of Information Management, v. 15, n. 5, p. 353-365, 1995.

cross ${ }^{\text {ref }}$

POPPO, L., \& ZENGER, T. Testing alternative theories of the firm: Transaction cost, knowledge-based, and measurement explanations for make-or-buy decisions in information services. Strategic Management Journal, v. 19, 853-877, 1998.

cross ${ }^{\text {ref }}$

RELATÓRIO FINAL TRENSURB Dimensionamento da Força de Trabalho na Trensurb - UFRGS, Porto Alegre, 2004.

TENG, J., CHEON, M., GROVER, V. Decisions to outsource information systems functions: Testing a strategytheoretic-discrepancy model. Decision Sciences, v. 26, n. 1, p. 75-103, 1995.

cross'

UZUMERI, M.; NEMBHARD, D. A Population of Learners: A New Way to Measure Organizational Learning. Journal of Operations Management, v. 16, p. 515-528, 1998.

crossef

WEIDENBAUM, M. Outsourcing: Pros and Cons, Business Horizons, Vol. 48, p. 311-315, 2005.

cross ${ }^{\text {ref }}$

\begin{abstract}
Choosing between outsourced or internal resources has been a capital question in several maintenance applications, mainly those relying on equipments of specific technology. Answers for such a question impact on the number and professional profile of workers assigned to tasks. We proposed a simplified approach aimed at defining a proper workload balance based on time and efficiency analysis. Data is collected from maintenance procedures and arranged by means of a relating matrix, which enables a better understanding of the time required for execution of procedures. The results are then combined with a new developed index that compares the times required by internal and outsource workers for task completion. Matters of availability are also taken into account. The method enables cost and performance comparison between internal and outsourced maintenance teams, and can be applied for hiring private and public resources, as well as for managing outsourced contracts. When applied in a public transportation company named TRENSURB S/A, the method identified the outsourced workers as the best option.
\end{abstract}

Keywords: Workforce balance, efficiency, maintenance, outsourcing.

\title{
Dados dos autores:
}

Nome completo: Diego Vinicius Souza de Souza

Filiação institucional: Universidade Federal do Rio Grande do Sul (UFRGS)

Departamento: Engenharia de Produção

Função ou cargo ocupado: Mestrando 
Endereço completo para correspondência: Avenida Osvaldo Aranha, 99, 5 andar, Porto Alegre, RS, Brasil, 90035-190

Telefones para contato: (51) 3308-3491

e-mail:vinicius@producao.ufrgs.br

Nome completo: Tiago Pascoal Filomena

Filiação institucional: The George Washington University

Departamento: Systems Engineering

Função ou cargo ocupado: doutorando

Endereço completo para correspondência: 8009 Eastern Avenue, apt. 302, MD, 20910, USA

Telefones para contato: 2024608628

e-mail: tiagopf@gwu.edu

Nome completo: Michel Jose Anzanello

Filiação institucional: RUTGERS - The State University of New Jersey

Departamento: Industrial and Systems Engineering

Função ou cargo ocupado: doutorando

Endereço completo para correspondência: 793 Bevier Road, Piscataway, NJ, 08854, USA

Telefones para contato: 7328016404

e-mail: michelja@eden.rutgers.edu

Nome completo: Francisco José Kliemann Neto

Filiação institucional: Universidade Federal do Rio Grande do Sul (UFRGS)

Departamento: Engenharia de Produção

Função ou cargo ocupado: Professor Adjunto

Endereço completo para correspondência: Avenida Osvaldo Aranha, 99, $5^{\circ}$ andar, Porto Alegre, RS, Brasil, 90035-190

Telefones para contato: (51) 3308-3491

e-mail:kliemann@producao.ufrgs.br 